\title{
Community Engaged Approaches to Cervical Cancer Prevention and Control in Sub-Saharan Africa: A Scoping Review Protocol
}

Magdiel A Habila ( $\triangle$ magdielhabila@email.arizona.edu )

University of Arizona https://orcid.org/0000-0003-4614-7772

Namoonga Mantina

University of Arizona

Linda Jepkoech Kimaru

University of Arizona

Jonah Musa

Jos University Teaching Hospital

Maia Ingram

Arizona State University

Atiene Sagay

Jos University Teaching Hospital

\section{Protocol}

Keywords: Cervical cancer prevention and control, community engagement, scoping review protocol, Africa

Posted Date: June 23rd, 2020

DOl: https://doi.org/10.21203/rs.3.rs-37012/v1

License: (c) (i) This work is licensed under a Creative Commons Attribution 4.0 International License.

Read Full License

Version of Record: A version of this preprint was published at Frontiers in Global Women's Health on July 19th, 2021. See the published version at https://doi.org/10.3389/fgwh.2021.697607. 


\section{Abstract}

\section{Introduction}

Cervical cancer is the fourth leading cause of death in women globally and remains the foremost cause of death in African women in spite of campaigns to increase education and prevention of the disease. The goal of participatory action research or community based participatory research is to engage communities in the research process in order to increase disease awareness and decrease disease mortality as a result of health disparities. The primary objective of this study is to summarize methods of community engagement in research on the prevention and control of cervical cancer in sub-Saharan Africa (SSA).

\section{Methods}

We will search through the following electronic databases for potential studies: PubMed, Embase, African Journals Online (AJOL), and African Index Medicus-WHO from inception until the date the last search will be conducted. Articles included will be restricted to those published in English and pertaining to research conducted in SSA. Articles generated from the search will be managed in our database. A narrative synthesis will be performed on the final list of included articles.

\section{Discussion}

This scoping review will provide evidence and perspectives on how research studies have engaged community members to bolster the cervical cancer prevention and control efforts. Findings of this review will contribute to the literature by identifying the benefits of community engagement in cervical cancer prevention in SSA and advance the science of cervical cancer prevention in the region and globally.

\section{Introduction}

Cervical cancer is the leading cause of death in African women, with an estimated 119,284 new cases in 2018 and an estimated 81,687 deaths in that year ${ }^{1,2}$. According to GLOBOCAN (2018) estimates, African women have the highest cancer incidence when compared to other continents (139.6 per 100,000), and they experience the highest mortality $(94.1 \text { per } 100,000)^{3}$. High mortality rates are primarily due to limited access to screening services and late presentation with diagnosis at advanced stages of the disease 4 . While studies have evaluated perceptions and knowledge about cervical cancer, few interventions have utilized participatory approaches to understand and address this disease burden in African communities.

For the purposes of this review, community engaged research is defined broadly as "research efforts that include community members in the development of a research question, interpretation of study results, 
and/ or implementation of research interventions or findings." ${ }^{5}$ Community based participatory research (CBPR), a well-known practice of community engagement, is a method for equal community engagement in the research process. The goal of this process is that research is driven by the needs of the community and that research findings benefit the community at the center of the research effort, resulting in translation of research to practice, and sustainability of research efforts ${ }^{6}$. The literature supports that underrepresented and underserved populations experience health disparities, and few strategies have been developed to eliminate these disparities ${ }^{7,8}$. CBPR is one tool that can be used within community settings to address disparities faced by the community ${ }^{9}$.

CBPR has many strengths, including that knowledge generated in the course of the study is relevant and useful to the community, that existing distrust of research can be replaced with trusting collaborative relationships between the community and researchers, and research findings are more likely to be translated into practice within the community ${ }^{6}$. However, the basis of community engaged research is that there is a relationship between the community members and the researcher that is intentionally built and maintained - sometimes even after the completion of the research project ${ }^{6}$ - making participatory research challenging when that relationship does not exist. Given the significant burden of cervical cancer on communities in Sub-Saharan Africa and the growing disparity in cervical cancer incidence between high income and low- and middle-income countries, it is extremely important to understand the ways that community engaged research practices have been used to address concerns about cervical cancer in atrisk communities in Sub-Saharan Africa.

There is evidence in the literature showing how CBPR has been used to increase knowledge about cancer screening in underserved populations. One study focused on increasing awareness about prostate cancer in African American men in Buffalo, New York. By developing community and institutional relationships, the researchers were able to recruit and educate men in this population about the importance of prostate cancer screening and made access to screening available for those who were interested. ${ }^{10}$ Another study focused on determining cervical cancer screening knowledge and correlates of cancer screening compliance among Haitian and Cuban immigrants to the United States living in Miami, Florida. These researchers employed CBPR because these communities are often stigmatized and are at high risk for cervical cancer due to their limited access to screening services. In collaboration with community health workers, the researchers were able to quantify the knowledge that these community members had about cervical cancer and HPV, enabling them to develop targeted interventions to serve the local Haitian and Cuban immigrant populations. ${ }^{11}$ These examples indicate that CBPR has been used to improve access to screening in various populations in the United States, and highlights this method's strengths at reaching medically underserved communities and addressing significant health disparities.

We will conduct a scoping review of the literature in order to: 1) highlight community engagement research efforts in the work of cervical cancer prevention and control in Sub-Saharan Africa (SSA), 2) describe the participatory approaches that have been employed to improve the effectiveness of cervical cancer prevention and control efforts in Sub-Saharan African communities, and 3) to determine best 
practices for community engagement in cervical cancer prevention and control. To our knowledge this is the first review that will evaluate cervical cancer prevention and control efforts from the lens of community engagement as an effective method of addressing health disparities in SSA.

\section{Methods}

This scoping review will be conducted and reported according to the Preferred Reporting Items for

Scoping Reviews. ${ }^{12}$ Scoping reviews are not eligible for registration. This review method will allow for the determination of the extent, range, and nature of community-based approaches that have been utilized for cervical cancer prevention and control in SSA.

\section{Data Sources}

The following electronic databases will be searched for identifying potential studies: PubMed, Embase, African Journals Online (AJOL), and African Index Medicus-WHO, from inception until the date the last search will be conducted. Reference lists of all primary studies and review articles will be checked for additional references. We will also search grey literature databases including: Health Management Information Consortium (HMIC) database, National Technical Information Service (NTIS) database, and OpenGrey. The search strategy will be developed with the assistance of a medical research librarian to include key search terms related to cervical cancer prevention and control, community engaged research practices including CBPR and community action research, and Sub-Saharan Africa. The primary studies of any previously published literature, systematic or scoping reviews will be evaluated to determine eligibility for inclusion. Literature search results will be combined and screened in Covidence (http://www.covidence.org) to remove duplicates.

\section{Study Selection Screening}

We will consider all study designs (experimental and observational) and study methods (qualitative and quantitative) for inclusion in this review. We will include studies that involve interventions that involve all types of community engagement in their research process with the goal of cervical cancer prevention and control. All studies included after the final literature search has been conducted will be added to our database of papers. Each study will be reviewed by two review authors. Disagreements will be resolved with discussion and consensus between the two reviewers with the help of a third reviewer if necessary. Review authors will continue with a two-stage screening process, title and abstract review, and full text review of the abstracts within the inclusion criteria. Results from the screening process will be reported in a PRISMA flow diagram.

Study Eligibility Criteria

Inclusion Criteria 
1. Studies involving cervical cancer (women who are at risk for cervical cancer or who have been diagnosed with cervical cancer)

2. Related to cervical cancer prevention or control.

3. Studies conducted in Sub-Saharan African countries

4. Studies that include a reference community engagement in research activities

5. No limitation on year of study publication

\section{Exclusion Criteria}

1. Studies not published in English

2. Articles reporting opinion information such as editorial reviews and commentaries

Study Population: Studies that include individuals at risk for cervical cancer in Sub-Saharan Africa will be considered for inclusion. Studies that focus on women at risk for cervical cancer including women who have a family history of cervical cancer, who are sexually active, who are using oral contraceptives, are of childbearing years, have reduced access to cervical screening services due to cost or distance from a health facility that offers these services, have not received the recommended dose of the human papillomavirus (HPV) vaccine, or are immunocompromised.

\section{Data Extraction}

A data extraction form will be developed in Microsoft Excel to guide data extract from the included studies to address the research objectives. The data to be extracted will include publication, location of the study, target population, description of intervention, description of the community engagement process employed, risk factors for inclusion in the study, and the outcome of the study. We will also outcome data if they are reported. We will use these data to synthesize the types of community based participatory interventions or programs that have been used in Sub-Saharan Africa for cervical cancer prevention, and to summarize which risk factors the community-based interventions were targeting.

\section{Discussion}

Despite numerous efforts to reduce risks for cervical cancer, an estimated 100,000 women are diagnosed with cervical cancer annually in SSA ${ }^{13}$. Approximately $62 \%$ of these women are expected to die from the disease without treatment ${ }^{13}$. This scoping review will highlight research that has engaged communities in order to bolster cervical cancer prevention and control efforts. We hope the findings of this scoping review will contribute to the literature by summarizing and detailing effects of community engagement in cervical cancer prevention in SSA, as well as highlighting best practices for engaging communities. 


\section{Abbreviations}

PRISMA-p: Preferred Reporting Items for Systematic Reviews and Meta-Analysis Protocols

SSA: Sub-Saharan Africa

CBPR: Community Based Participatory Research

CAR: Community Action Research

\section{Declarations}

\section{Ethics Approval and Consent to Participate}

Not applicable.

\section{Consent for Publication}

Not applicable.

\section{Availability of Data and Materials}

Not applicable.

\section{Competing Interests}

The authors declare that they have no competing interests.

\section{Funding}

None.

\section{Authors Contributions}

$\mathrm{MH}$ conceptualized the study under the supervision of $\mathrm{Ml}$, with further guidance from JM and AS who have experience in cervical cancer prevention and control in SSA. MH, JK, and NM contributed to the writing of the proposal. All authors reviewed and approved the final draft of the proposal.

\section{Acknowledgements}

The authors would like to thank Jean McClelland for the technical assistance in developing the search strategy and Mel and Enid Zuckerman College of Public Health at the University of Arizona for the provision of resources for this review.

\section{References}


1. WHO IARC. Cancer Fact Sheets: Cervix uteri. Globocan 2018. 2018;876:6-7. http://gco.iarc.fr/today.

2. Arbyn $M$, Weiderpass $E$, Bruni $L$, et al. Estimates of incidence and mortality of cervical cancer in 2018 : a worldwide analysis. Lancet Glob Heal. 2020;8(2):e191-e203. doi:10.1016/S2214-109X(19)30482-6

3. Bray F, Ferlay J, Soerjomataram I, Siegel RL, Torre LA, Jemal A. Global cancer statistics 2018: GLOBOCAN estimates of incidence and mortality worldwide for 36 cancers in 185 countries. $C A$ Cancer J Clin. 2018;68(6):394-424. doi:10.3322/caac.21492

4. Torre LA, Siegel RL, Ward EM, Jemal A. Global cancer incidence and mortality rates and trends - An update. Cancer Epidemiol Biomarkers Prev. 2016;25(1):16-27. doi:10.1158/1055-9965.EPI-15-0578

5. Lavery SH, Smith ML, Esparza AA, Hrushow A, Moore M, Reed DF. The community action model: A community-driven model designed to address disparities in health. Am J Public Health. 2005;95(4):611-616. doi:10.2105/AJPH.2004.047704

6. Israel BA, Schulz AJ, Parker EA, Becker AB. REVIEW OF COMMUNITY-BASED RESEARCH: Assessing Partnership Approaches to Improve Public Health. Annu Rev Public Health. 1998;19(1):173-202. doi:10.1146/annurev.publhealth.19.1.173

7. Xia R, Stone JR, Hoffman JE, Klappa SG. Promoting Community Health and Eliminating Health Disparities Through Community-Based Participatory Research. Phys Ther. 2016;96(3):410-417. doi:10.2522/ptj.20140529

8. Azim HA, Nguyen B, Brohée S, Zoppoli G, Sotiriou C. Genomic aberrations in young and elderly breast cancer patients. BMC Med. 2015;13(1). doi:10.1186/s12916-015-0504-3

9. Wallerstein N, Duran B. Community-based participatory research contributions to intervention research: The intersection of science and practice to improve health equity. Am J Public Health. 2010;100(SUPPL. 1):40-46. doi:10.2105/AJPH.2009.184036

10. Ross L, Jarrett J, Smallwood S, et al. Using CBPR to Extend Prostate Cancer Education, Counseling and Screening Opportunities to Urban-Dwelling African Americans. J Cancer Educ. 2017;176(12):139148. doi:10.1016/j.physbeh.2017.03.040

11. Seay JS, Carrasquillo O, Campos NG, et al. Cancer screening utilization among immigrant women in Miami, Florida. Prog Community Heal Partnerships Res Educ Action. 2015;9:9. doi:10.1353/cpr.2015.0027

12. Tricco AC, Lillie E, Zarin W, et al. PRISMA extension for scoping reviews (PRISMA-ScR): Checklist and explanation. Ann Intern Med. 2018;169(7):467-473. doi:10.7326/M18-0850

13. Media U. UNAIDS and IAEA Forge a Powerful Partnership Against the Interlinked Disease of Cervical Cancer and HIV.https://www.unaids.org/en/cervical_cancer. Published February 7, 2020.

\section{Supplementary Files}

This is a list of supplementary files associated with this preprint. Click to download.

- BMCSystematicReviewsCoverLetter.docx 